\title{
Improved limit to the diffuse flux of ultrahigh energy neutrinos from the Pierre Auger Observatory
}

A. Aab, ${ }^{1}$ P. Abreu, ${ }^{2}$ M. Aglietta, ${ }^{3}$ E. J. Ahn, ${ }^{4}$ I. Al Samarai, ${ }^{5}$ I. F. M. Albuquerque, ${ }^{6}$ I. Allekotte, ${ }^{7}$ P. Allison, ${ }^{8}$ A. Almela,${ }^{9,10}$ J. Alvarez Castillo, ${ }^{11} \mathrm{~J}$. Alvarez-Muñiz, ${ }^{12}$ R. Alves Batista, ${ }^{13}$ M. Ambrosio, ${ }^{14}$ A. Aminaei, ${ }^{15}$ L. Anchordoqui, ${ }^{16}$ S. Andringa, ${ }^{2}$ C. Aramo, ${ }^{14}$ V. M. Aranda,${ }^{17}$ F. Arqueros, ${ }^{17}$ N. Arsene, ${ }^{18}$ H. Asorey,${ }^{7,19}$ P. Assis, ${ }^{2}$ J. Aublin, ${ }^{20}$ M. Ave, ${ }^{7}$ M. Avenier, ${ }^{21}$ G. Avila, ${ }^{22}$ N. Awal, ${ }^{23}$ A. M. Badescu, ${ }^{24}$ K. B. Barber, ${ }^{25}$ J. Bäuml, ${ }^{26}$ C. Bauss ${ }^{26}$ J. J. Beatty, ${ }^{8}$ K. H. Becker, ${ }^{27}$ J. A. Bellido, ${ }^{25}$ C. Berat, ${ }^{21}$ M. E. Bertaina, ${ }^{3}$ X. Bertou, ${ }^{7}$ P. L. Biermann, ${ }^{28}$ P. Billoir, ${ }^{20}$ S. G. Blaess, ${ }^{25}$ A. Blanco, ${ }^{2}$ M. Blanco, ${ }^{20}$ C. Bleve, ${ }^{29}$ H. Blümer, ${ }^{26,30}$ M. Boháčová, ${ }^{31}$ D. Boncioli, ${ }^{32}$ C. Bonifazi, ${ }^{33}$ N. Borodai,${ }^{34} \mathrm{~J}$. Brack,${ }^{35} \mathrm{I}$. Brancus,${ }^{36}$ A. Bridgeman ${ }^{30}$ P. Brogueira, ${ }^{2}$ W. C. Brown,${ }^{37}$ P. Buchholz, ${ }^{1}$ A. Bueno, ${ }^{38}$ S. Buitink, ${ }^{15}$ M. Buscemi,${ }^{14}$

K. S. Caballero-Mora, ${ }^{39}$ B. Caccianiga ${ }^{40}$ L. Caccianiga, ${ }^{20}$ M. Candusso, ${ }^{41}$ L. Caramete ${ }^{42}$ R. Caruso, ${ }^{43}$ A. Castellina, ${ }^{3}$

G. Cataldi, ${ }^{29}$ L. Cazon, ${ }^{2}$ R. Cester ${ }^{44}$ A. G. Chavez,${ }^{45}$ A. Chiavassa, ${ }^{3}$ J. A. Chinellato, ${ }^{46}$ J. Chudoba ${ }^{31}$ M. Cilmo, ${ }^{14}$ R. W. Clay, ${ }^{25}$ G. Cocciolo, ${ }^{29}$ R. Colalillo, ${ }^{14}$ A. Coleman,${ }^{47}$ L. Collica, ${ }^{40}$ M. R. Coluccia ${ }^{29}$ R. Conceição, ${ }^{2}$ F. Contreras,${ }^{48}$ M. J. Cooper, ${ }^{25}$ A. Cordier, ${ }^{49}$ S. Coutu, ${ }^{47}$ C. E. Covault,${ }^{50}$ J. Cronin, ${ }^{51}$ R. Dallier, ${ }^{52,53}$ B. Daniel,${ }^{46}$ S. Dasso, ${ }^{54,55}$ K. Daumiller, ${ }^{30}$ B. R. Dawson, ${ }^{25}$ R. M. de Almeida ${ }^{56}$ S. J. de Jong, ${ }^{15,57}$ G. De Mauro, ${ }^{15}$ J. R. T. de Mello Neto, ${ }^{33}$ I. De Mitri, ${ }^{29}$ J. de Oliveira, ${ }^{56}$ V. de Souza, ${ }^{58}$ L. del Peral,${ }^{59}$ O. Deligny, ${ }^{5}$ H. Dembinski, ${ }^{30}$ N. Dhital,${ }^{60}$ C. Di Giulio,${ }^{41}$ A. Di Matteo, ${ }^{61}$ J. C. Diaz ${ }^{60}$ M. L. Díaz Castro, ${ }^{46}$ F. Diogo, ${ }^{2}$ C. Dobrigkeit, ${ }^{46}$ W. Docters, ${ }^{62}$ J. C. D'Olivo,${ }^{11}$ A. Dorofeev, ${ }^{35}$ Q. Dorosti Hasankiadeh ${ }^{30}$ M. T. Dova,${ }^{63}$ J. Ebr, ${ }^{31}$ R. Engel, ${ }^{30}$ M. Erdmann, ${ }^{64}$ M. Erfani, ${ }^{1}$ C. O. Escobar, ${ }^{4,46}$ J. Espadanal, ${ }^{2}$ A. Etchegoyen, ${ }^{10,9}$ H. Falcke, ${ }^{15,65,57}$ K. Fang, ${ }^{51}$ G. Farrar, ${ }^{23}$ A. C. Fauth ${ }^{46}$ N. Fazzini, ${ }^{4}$ A. P. Ferguson,${ }^{50}$ M. Fernandes,${ }^{33}$ B. Fick,${ }^{60}$ J. M. Figueira, ${ }^{10}$ A. Filevich, ${ }^{10}$ A. Filipčič ${ }^{66,67}$ B. D. Fox, ${ }^{68}$ O. Fratu, ${ }^{24}$ M. M. Freire, ${ }^{69}$ B. Fuchs,${ }^{26}$ T. Fujiii, ${ }^{51}$ B. García, ${ }^{70}$ D. Garcia-Pinto, ${ }^{17}$ F. Gate, ${ }^{52}$ H. Gemmeke, ${ }^{71}$ A. Gherghel-Lascu, ${ }^{36}$ P. L. Ghia, ${ }^{20}$ U. Giaccari, ${ }^{33}$ M. Giammarchi, ${ }^{40}$ M. Giller ${ }^{72}$ D. Głas ${ }^{72}$ C. Glaser ${ }^{64}$ H. Glass, ${ }^{4}$ G. Golup ${ }^{7}$ M. Gómez Berisso, ${ }^{7}$ P. F. Gómez Vitale, ${ }^{22}$ N. González, ${ }^{10}$ B. Gookin, ${ }^{35}$ J. Gordon, ${ }^{8}$ A. Gorgi, ${ }^{3}$ P. Gorham ${ }^{6}{ }^{8}$ P. Gouffon, ${ }^{6}$ N. Griffith, ${ }^{8}$ A. F. Grillo, ${ }^{32}$ T. D. Grubb, ${ }^{25}$ Y. Guardincerri, ${ }^{55,}$ F. Guarino, ${ }^{14}$ G. P. Guedes, ${ }^{73}$ M. R. Hampel, ${ }^{10}$ P. Hansen,${ }^{63}$ D. Harari, ${ }^{7}$ T. A. Harrison,${ }^{25}$ S. Hartmann,${ }^{64}$ J. L. Harton, ${ }^{35}$ A. Haungs,${ }^{30}$ T. Hebbeker, ${ }^{64}$ D. Heck,${ }^{30}$ P. Heimann, ${ }^{1}$ A. E. Herve,${ }^{30}$ G. C. Hill,,${ }^{25}$ C. Hojvat, ${ }^{4}$ N. Hollon, ${ }^{51}$ E. Holt ${ }^{30}$ P. Homola, ${ }^{27}$ J. R. Hörandel, ${ }^{15,57}$ P. Horvath, ${ }^{74}$ M. Hrabovský, ${ }^{74,31}$ D. Huber ${ }^{26}$ T. Huege, ${ }^{30}$ A. Insolia, ${ }^{43}$ P. G. Isar, ${ }^{42}$ I. Jandt, ${ }^{27}$ S. Jansen, ${ }^{15,57}$ C. Jarne ${ }^{63}$ J. A. Johnsen,${ }^{75}$ M. Josebachuili, ${ }^{10}$ A. Kääpä, ${ }^{27}$ O. Kambeitz, ${ }^{26}$ K. H. Kampert, ${ }^{27}$ P. Kasper, ${ }^{4}$ I. Katkov ${ }^{26}$ B. Kégl, ${ }^{49}$ B. Keilhauer, ${ }^{30}$ A. Keivani, ${ }^{47}$ E. Kemp, ${ }^{46}$ R. M. Kieckhafer ${ }^{60}$ H. O. Klages, ${ }^{30}$ M. Kleifges, ${ }^{71}$ J. Kleinfeller, ${ }^{48}$ R. Krause, ${ }^{64}$ N. Krohm,${ }^{27}$ O. Krömer ${ }^{71}$ D. Kuempel, ${ }^{64}$ N. Kunka, ${ }^{71}$ D. LaHurd, ${ }^{50}$ L. Latronico, ${ }^{3}$ R. Lauer, ${ }^{76}$ M. Lauscher, ${ }^{64}$ P. Lautridou, ${ }^{52}$ S. Le Coz,${ }^{21}$ D. Lebrun ${ }^{21}$ P. Lebrun, ${ }^{4}$ M. A. Leigui de Oliveira, ${ }^{77}$ A. Letessier-Selvon, ${ }^{20}$ I. Lhenry-Yvon, ${ }^{5}$ K. Link, ${ }^{26}$ L. Lopes, ${ }^{2}$ R. López,${ }^{78}$ A. López Casado, ${ }^{12}$ K. Louedec, ${ }^{21}$ L. Lu, ${ }^{27,79}$ A. Lucero, ${ }^{10}$ M. Malacari, ${ }^{25}$ S. Maldera, ${ }^{3}$ M. Mallamaci, ${ }^{40}$ J. Maller, ${ }^{52}$ D. Mandat, ${ }^{31}$ P. Mantsch, A. G. Mariazzi, ${ }^{63}$ V. Marin ${ }^{52}$ I. C. Mariş, ${ }^{38}$ G. Marsella, ${ }^{29}$ D. Martello, ${ }^{29}$ L. Martin,${ }^{52,53}$ H. Martinez,${ }^{80}$ O. Martínez Bravo, ${ }^{78}$ D. Martraire, ${ }^{5}$ J. J. Masías Meza, ${ }^{55}$ H. J. Mathes,${ }^{30}$ S. Mathys, ${ }^{27}$ J. Matthews, ${ }^{81}$ J. A. J. Matthews, ${ }^{76}$ G. Matthiae, ${ }^{41}$ D. Maurel,,${ }^{26}$ D. Maurizio, ${ }^{82}$ E. Mayotte, ${ }^{75}$ P. O. Mazur, ${ }^{4}$ C. Medina, ${ }^{75}$ G. Medina-Tanco, ${ }^{11}$ R. Meissner, ${ }^{64}$ V. B. B. Mello, ${ }^{33}$ D. Melo, ${ }^{10}$ A. Menshikov, ${ }^{71}$ S. Messina, ${ }^{62}$ R. Meyhandan, ${ }^{68}$ M. I. Micheletti, ${ }^{69}$ L. Middendorf, ${ }^{64}$ I. A. Minaya, ${ }^{17}$ L. Miramonti, ${ }^{40}$ B. Mitrica ${ }^{36}$ L. Molina-Bueno, ${ }^{38}$ S. Mollerach, ${ }^{7}$ F. Montanet,${ }^{21}$ C. Morello, ${ }^{3}$ M. Mostafá, ${ }^{47}$ C. A. Moura ${ }^{77}$ M. A. Muller, ${ }^{46,83}$ G. Müller ${ }^{64}$ S. Müller, ${ }^{30}$ R. Mussa, ${ }^{44}$ G. Navarra, ${ }^{3,}$ J. L. Navarro, ${ }^{38, \$}$ S. Navas, ${ }^{38}$ P. Necesal, ${ }^{31}$ L. Nellen, ${ }^{11}$ A. Nelles, ${ }^{15,57}$ J. Neuser, ${ }^{27}$ P. H. Nguyen, ${ }^{25}$ M. Niculescu-Oglinzanu, ${ }^{36}$ M. Niechciol, ${ }^{1}$ L. Niemietz, ${ }^{27}$ T. Niggemann, ${ }^{64}$ D. Nitz ${ }^{60}$ D. Nosek,${ }^{84}$ V. Novotny,${ }^{84}$ L. Nožka, ${ }^{74}$ L. Ochilo, ${ }^{1}$ F. Oikonomou ${ }^{47}$ A. Olinto, ${ }^{51}$ N. Pacheco, ${ }^{59}$

D. Pakk Selmi-Dei ${ }^{46}$ M. Palatka, ${ }^{31}$ J. Pallotta ${ }^{85}$ P. Papenbreer ${ }^{27}$ G. Parente, ${ }^{12}$ A. Parra, ${ }^{78}$ T. Paul, ${ }^{16,86}$ M. Pech, ${ }^{31}$ J. Pękala ${ }^{34}$ R. Pelayo, ${ }^{87}$ I. M. Pepe,${ }^{88}$ L. Perrone ${ }^{29}$ E. Petermann, ${ }^{89}$ C. Peters,${ }^{64}$ S. Petrera, ${ }^{61,90}$ Y. Petrov,${ }^{35}$ J. Phuntsok, ${ }^{47}$ R. Piegaia, ${ }^{55}$ T. Pierog, ${ }^{30}$ P. Pieroni,${ }^{55}$ M. Pimenta, ${ }^{2}$ V. Pirronello, ${ }^{43}$ M. Platino, ${ }^{10}$ M. Plum, ${ }^{64}$ A. Porcelli,${ }^{30}$ C. Porowski ${ }^{34}$

R. R. Prado, ${ }^{58}$ P. Privitera, ${ }^{51}$ M. Prouza, ${ }^{31}$ V. Purrello, ${ }^{7}$ E. J. Quel, ${ }^{85}$ S. Querchfeld ${ }^{27}$ S. Quinn, ${ }^{50}$ J. Rautenberg, ${ }^{27}$ O. Ravel,${ }^{52}$ D. Ravignani, ${ }^{10}$ B. Revenu, ${ }^{52}$ J. Ridky, ${ }^{31}$ S. Riggi, ${ }^{43}$ M. Risse, ${ }^{1}$ P. Ristori, ${ }^{85}$ V. Rizi,${ }^{61}$

W. Rodrigues de Carvalho, ${ }_{17}$ G. Rodriguez Fernandez, ${ }^{41}$ J. Rodriguez Rojo ${ }^{48}$ M. D. Rodríguez-Frías, ${ }^{59}$ D. Rogozin ${ }^{30}$ J. Rosado, ${ }^{17}$ M. Roth, ${ }^{30}$ E. Roulet, ${ }^{7}$ A. C. Rovero, ${ }^{54}$ S. J. Saffi, ${ }^{25}$ A. Saftoiu, ${ }^{36}$ F. Salamida, ${ }^{5}$ H. Salazar, ${ }^{78}$ A. Saleh, ${ }^{67}$ F. Salesa Greus, ${ }^{47}$ G. Salina, ${ }^{41}$ F. Sánchez, ${ }^{10}$ P. Sanchez-Lucas, ${ }^{38}$ E. Santos ${ }^{46}$ E. M. Santos, ${ }^{6}$ F. Sarazin,${ }^{75}$ B. Sarkar, ${ }^{27}$ R. Sarmento, ${ }^{2}$ R. Sato,${ }^{48}$ C. Scarso, ${ }^{48}$ M. Schauer, ${ }^{27}$ V. Scherini ${ }^{29}$ H. Schieler, ${ }^{30}$ P. Schiffer, ${ }^{13}$ D. Schmidt, ${ }^{30}$ O. Scholten,${ }^{62,}$ H. Schoorlemmer, ${ }^{68}$ P. Schovánek, ${ }^{31}$ F. G. Schöder, ${ }^{30}$ A. Schulz, ${ }^{30}$ J. Schulz, ${ }^{15}$ J. Schumacher, ${ }^{64}$ S. J. Sciutto, ${ }^{63}$

A. Segreto ${ }^{91}$ M. Settimo, ${ }^{20}$ A. Shadkam,${ }^{81}$ R. C. Shellard, ${ }^{82}$ I. Sidelnik, ${ }^{7}$ G. Sigl, ${ }^{13}$ O. Sima, ${ }^{18}$ A. Śmiałkowski, ${ }^{72}$ R. Šmída, ${ }^{30}$ G. R. Snow ${ }^{89}$ P. Sommers, ${ }^{47}$ J. Sorokin, ${ }^{25}$ R. Squartini, ${ }^{48}$ Y. N. Srivastava, ${ }^{86}$ D. Stanca, ${ }^{36}$ S. Stanič ${ }^{67}$ J. Stapleton, ${ }^{8}$ J. Stasielak ${ }^{34}$ M. Stephan, ${ }^{64}$ A. Stutz ${ }^{21}$ F. Suarez,${ }^{10}$ T. Suomijärvi ${ }^{5}$ A. D. Supanitsky, ${ }^{54}$ M. S. Sutherland, ${ }^{8}$ J. Swain, ${ }^{86}$ Z. Szadkowski, ${ }^{72}$ O. A. Taborda, ${ }^{7}$ A. Tapia, ${ }^{10}$ A. Tepe, ${ }^{1}$ V. M. Theodoro, ${ }^{46}$ J. Tiffenberg, ${ }^{55,}$ C. Timmermans, ${ }^{57,15}$ 
C. J. Todero Peixoto, ${ }^{92}$ G. Toma,${ }^{36}$ L. Tomankova,${ }^{30}$ B. Tomé, ${ }^{2}$ A. Tonachini,${ }^{44}$ G. Torralba Elipe,${ }^{12}$ D. Torres Machado, ${ }^{33}$ P. Travnicek, ${ }^{31}$ R. Ulrich ${ }^{30}$ M. Unger ${ }^{23}$ M. Urban,${ }^{64}$ J. F. Valdés Galicia, ${ }^{11}$ I. Valiño, ${ }^{12}$ L. Valore, ${ }^{14}$ G. van Aar, ${ }^{15}$ P. van Bodegom, ${ }^{25}$ A. M. van den Berg, ${ }^{62}$ S. van Velzen, ${ }^{15}$ A. van Vliet,${ }^{13}$ E. Varela,${ }^{78}$ B. Vargas Cárdenas, ${ }^{11}$ G. Varner,${ }^{68}$ R. Vasquez, ${ }^{33}$ J. R. Vázquez, ${ }^{17}$ R. A. Vázquez, ${ }^{12}$ D. Veberičc,${ }^{30}$ V. Verzi, ${ }^{41} \mathrm{~J}$. Vicha, ${ }^{31}$ M. Videla, ${ }^{10}$ L. Villaseñor, ${ }^{45}$ B. Vlcek, ${ }^{59}$ S. Vorobiov ${ }^{67}$ H. Wahlberg, ${ }^{63}$ O. Wainberg, ${ }^{109}$ D. Walz ${ }^{64}$ A. A. Watson, ${ }^{79}$ M. Weber, ${ }^{71}$ K. Weidenhaupt ${ }^{64}$ A. Weindl,${ }^{30}$ F. Werner, ${ }^{26}$ A. Widom, ${ }^{86}$ L. Wiencke, ${ }^{75}$ H. Wilczyński, ${ }^{34}$ T. Winchen, ${ }^{27}$ D. Wittkowski, ${ }^{27}$ B. Wundheiler, ${ }^{10}$ S. Wykes, ${ }^{15}$ L. Yang, ${ }^{67}$ T. Yapici, ${ }^{60}$ A. Yushkov, ${ }^{1}$ E. Zas, ${ }^{12}$ D. Zavrtanik, ${ }^{67,66}$ M. Zavrtanik, ${ }^{66,67}$ A. Zepeda, ${ }^{80}$ Y. Zhu, ${ }^{71}$ B. Zimmermann, ${ }^{71}$ M. Ziolkowski, ${ }^{1}$ and F. Zuccarello ${ }^{43}$

(Pierre Auger Collaboration) ${ }^{\text {ๆ }}$

\author{
${ }^{1}$ Universität Siegen, Siegen, Germany \\ ${ }^{2}$ Laboratório de Instrumentação e Física Experimental de Partículas-LIP and Instituto Superior \\ Técnico-IST, Universidade de Lisboa-UL, Portugal \\ ${ }^{3}$ Osservatorio Astrofisico di Torino (INAF), Università di Torino and Sezione INFN, \\ Torino, Italy \\ ${ }^{4}$ Fermilab, Batavia, Illinois, USA \\ ${ }^{5}$ Institut de Physique Nucléaire d'Orsay (IPNO), Université Paris 11, CNRS-IN2P3, Orsay, France \\ ${ }^{6}$ Universidade de São Paulo, Instituto de Física, São Paulo, SP, Brazil \\ ${ }^{7}$ Centro Atómico Bariloche and Instituto Balseiro (CNEA-UNCuyo-CONICET), \\ San Carlos de Bariloche, Argentina \\ ${ }^{8}$ Ohio State University, Columbus, Ohio, USA \\ ${ }^{9}$ Universidad Tecnológica Nacional-Facultad Regional Buenos Aires, Buenos Aires, Argentina \\ ${ }^{10}$ Instituto de Tecnologías en Detección y Astropartículas (CNEA, CONICET, UNSAM), \\ Buenos Aires, Argentina \\ ${ }^{11}$ Universidad Nacional Autónoma de Mexico, Mexico, D.F., Mexico \\ ${ }^{12}$ Universidad de Santiago de Compostela, Spain \\ ${ }^{13}$ Universität Hamburg, Hamburg, Germany \\ ${ }^{14}$ Università di Napoli "Federico II" and Sezione INFN, Napoli, Italy \\ ${ }^{15}$ IMAPP, Radboud University Nijmegen, Netherlands \\ ${ }^{16}$ Department of Physics and Astronomy, Lehman College, City University of New York, New York, USA \\ ${ }^{17}$ Universidad Complutense de Madrid, Madrid, Spain \\ ${ }^{18}$ University of Bucharest, Physics Department, Romania \\ ${ }^{19}$ Universidad Industrial de Santander, Colombia \\ ${ }^{20}$ Laboratoire de Physique Nucléaire et de Hautes Energies (LPNHE), Universités Paris 6 et Paris 7 , \\ CNRS-IN2P3, Paris, France \\ ${ }^{21}$ Laboratoire de Physique Subatomique et de Cosmologie (LPSC), Université Grenoble-Alpes, \\ CNRS/IN2P3, France \\ ${ }^{22}$ Observatorio Pierre Auger and Comisión Nacional de Energía Atómica, Malargüe, Argentina \\ ${ }^{23}$ New York University, New York, New York, USA \\ ${ }^{24}$ University Politehnica of Bucharest, Romania \\ ${ }^{25}$ University of Adelaide, Adelaide, S.A., Australia \\ ${ }^{26}$ Karlsruhe Institute of Technology-Campus South-Institut für Experimentelle Kernphysik (IEKP), \\ Karlsruhe, Germany \\ ${ }^{27}$ Bergische Universität Wuppertal, Wuppertal, Germany \\ ${ }^{28}$ Max-Planck-Institut für Radioastronomie, Bonn, Germany \\ ${ }^{29}$ Dipartimento di Matematica e Fisica "E. De Giorgi" dell'Università del Salento and Sezione INFN, \\ Lecce, Italy \\ ${ }^{30}$ Karlsruhe Institute of Technology-Campus North-Institut für Kernphysik, \\ Karlsruhe, Germany \\ ${ }^{31}$ Institute of Physics of the Academy of Sciences of the Czech Republic, \\ Prague, Czech Republic \\ ${ }^{32}$ INFN, Laboratori Nazionali del Gran Sasso, Assergi (L'Aquila), Italy \\ ${ }^{33}$ Universidade Federal do Rio de Janeiro, Instituto de Física, Rio de Janeiro, RJ, Brazil \\ ${ }^{34}$ Institute of Nuclear Physics PAN, Krakow, Poland \\ ${ }^{35}$ Colorado State University, Fort Collins, Colorado, USA \\ 36 'Horia Hulubei' National Institute for Physics and Nuclear Engineering, Bucharest-Magurele, Romania \\ ${ }^{37}$ Colorado State University, Pueblo, Colorado, USA \\ ${ }^{38}$ Universidad de Granada and C.A.F.P.E., Granada, Spain \\ ${ }^{39}$ Universidad Autónoma de Chiapas, Tuxtla Gutiérrez, Chiapas, Mexico
}


${ }^{40}$ Università di Milano and Sezione INFN, Milan, Italy

${ }^{41}$ Università di Roma II "Tor Vergata" and Sezione INFN, Roma, Italy

${ }^{42}$ Institute of Space Sciences, Bucharest, Romania

${ }^{43}$ Università di Catania and Sezione INFN, Catania, Italy

${ }^{44}$ Università di Torino and Sezione INFN, Torino, Italy

${ }^{45}$ Universidad Michoacana de San Nicolás de Hidalgo,

Morelia, Michoacán, Mexico

${ }^{46}$ Universidade Estadual de Campinas, IFGW, Campinas, SP, Brazil

${ }^{47}$ Pennsylvania State University, University Park, USA

${ }^{48}$ Observatorio Pierre Auger, Malargüe, Argentina

${ }^{49}$ Laboratoire de l'Accélérateur Linéaire (LAL), Université Paris 11, CNRS-IN2P3, Orsay, France

${ }^{50}$ Case Western Reserve University, Cleveland, Ohio, USA

${ }^{51}$ University of Chicago, Enrico Fermi Institute, Chicago, Illinois, USA

${ }^{52}$ SUBATECH, École des Mines de Nantes, CNRS-IN2P3, Université de Nantes, Nantes, France

${ }^{53}$ Station de Radioastronomie de Nançay, Observatoire de Paris, CNRS/INSU, Nançay, France

${ }^{54}$ Instituto de Astronomía y Física del Espacio (IAFE, CONICET-UBA), Buenos Aires, Argentina

${ }^{55}$ Departamento de Física, FCEyN, Universidad de Buenos Aires and CONICET, Argentina

${ }^{56}$ Universidade Federal Fluminense, EEIMVR, Volta Redonda, RJ, Brazil

${ }^{57}$ Nikhef, Science Park, Amsterdam, Netherlands

${ }^{58}$ Universidade de São Paulo, Instituto de Física de São Carlos, São Carlos, SP, Brazil

${ }^{59}$ Universidad de Alcalá, Alcalá de Henares, Spain

${ }^{60}$ Michigan Technological University, Houghton, Michigan, USA

${ }^{61}$ Dipartimento di Scienze Fisiche e Chimiche dell'Università dell'Aquila and INFN, Italy

${ }^{62}$ KVI-Center for Advanced Radiation Technology, University of Groningen, Groningen, Netherlands

${ }^{63}$ IFLP, Universidad Nacional de La Plata and CONICET, La Plata, Argentina

${ }^{64}$ RWTH Aachen University, III. Physikalisches Institut A, Aachen, Germany

${ }^{65}$ ASTRON, Dwingeloo, Netherlands

${ }^{66}$ Experimental Particle Physics Department, J. Stefan Institute, Ljubljana, Slovenia

${ }^{67}$ Laboratory for Astroparticle Physics, University of Nova Gorica, Slovenia

${ }^{68}$ University of Hawaii, Honolulu, Hawaii, USA

${ }^{69}$ Instituto de Física de Rosario (IFIR)-CONICET/U.N.R. and Facultad de Ciencias Bioquímicas y Farmacéuticas U.N.R., Rosario, Argentina

${ }^{70}$ Instituto de Tecnologías en Detección y Astropartículas (CNEA, CONICET, UNSAM), and Universidad Tecnológica Nacional-Facultad Regional Mendoza (CONICET/CNEA),

Mendoza, Argentina

${ }^{71}$ Karlsruhe Institute of Technology-Campus North-Institut für Prozessdatenverarbeitung und Elektronik, Germany

${ }^{72}$ University of Łódź, Łódź, Poland

${ }^{73}$ Universidade Estadual de Feira de Santana, Brazil

${ }^{74}$ Palacky University, RCPTM, Olomouc, Czech Republic

${ }^{75}$ Colorado School of Mines, Golden, Colorado, USA

${ }^{76}$ University of New Mexico, Albuquerque, New Mexico, USA

${ }^{77}$ Universidade Federal do ABC, Santo André, SP, Brazil

${ }^{78}$ Benemérita Universidad Autónoma de Puebla, Mexico

${ }^{79}$ School of Physics and Astronomy, University of Leeds, United Kingdom

${ }^{80}$ Centro de Investigación y de Estudios Avanzados del IPN (CINVESTAV), Mexico, D.F., Mexico

${ }^{81}$ Louisiana State University, Baton Rouge, Louisiana, USA

${ }^{82}$ Centro Brasileiro de Pesquisas Fisicas, Rio de Janeiro, RJ, Brazil

${ }^{83}$ Universidade Federal de Pelotas, Pelotas, RS, Brazil

${ }^{84}$ Charles University, Faculty of Mathematics and Physics, Institute of Particle and Nuclear Physics, Prague, Czech Republic

${ }^{85}$ Centro de Investigaciones en Láseres y Aplicaciones, CITEDEF and CONICET, Argentina

${ }^{86}$ Northeastern University, Boston, Massachusetts, USA

${ }^{87}$ Unidad Profesional Interdisciplinaria en Ingeniería y Tecnologías Avanzadas del Instituto Politécnico Nacional (UPIITA-IPN), Mexico, D.F., Mexico

${ }^{88}$ Universidade Federal da Bahia, Salvador, BA, Brazil

${ }^{89}$ University of Nebraska, Lincoln, Nebraska, USA

${ }^{90}$ Gran Sasso Science Institute (INFN), L'Aquila, Italy 
${ }^{91}$ Istituto di Astrofisica Spaziale e Fisica Cosmica di Palermo (INAF), Palermo, Italy

${ }^{92}$ Universidade de São Paulo, Escola de Engenharia de Lorena, Lorena, SP, Brazil

(Received 18 March 2015; published 26 May 2015)

Neutrinos in the cosmic ray flux with energies near $1 \mathrm{EeV}$ and above are detectable with the Surface Detector array (SD) of the Pierre Auger Observatory. We report here on searches through Auger data from 1 January 2004 until 20 June 2013. No neutrino candidates were found, yielding a limit to the diffuse flux of ultrahigh energy neutrinos that challenges the Waxman-Bahcall bound predictions. Neutrino identification is attempted using the broad time structure of the signals expected in the SD stations, and is efficiently done for neutrinos of all flavors interacting in the atmosphere at large zenith angles, as well as for "Earth-skimming" neutrino interactions in the case of tau neutrinos. In this paper the searches for downward-going neutrinos in the zenith angle bins $60^{\circ}-75^{\circ}$ and $75^{\circ}-90^{\circ}$ as well as for upward-going neutrinos, are combined to give a single limit. The $90 \%$ C.L. single-flavor limit to the diffuse flux of ultrahigh energy neutrinos with an $E^{-2}$ spectrum in the energy range $1.0 \times 10^{17} \mathrm{eV}-2.5 \times 10^{19} \mathrm{eV}$ is $E_{\nu}^{2} d N_{\nu} / d E_{\nu}<6.4 \times 10^{-9} \mathrm{GeV} \mathrm{cm}^{-2} \mathrm{~s}^{-1} \mathrm{sr}^{-1}$.

\section{INTRODUCTION}

The flux of ultrahigh energy cosmic rays (UHECRs) above $\sim 5 \times 10^{19} \mathrm{eV}$ is known to be suppressed with respect to that extrapolated from lower energies. This feature has been seen in the UHECR spectrum [1,2], with the position of the break being compatible with the Greisen-Zatsepin-Kuzmin effect [3], i.e. the interaction of UHECRs with the cosmic microwave background $(\mathrm{CMB})$ radiation. However, other explanations are possible, most prominently a scenario where the limiting energy of the UHECR sources is being observed [4]. Key to distinguishing between these two scenarios is the determination of the composition of the UHECRs [5,6], with the second scenario predicting increasing fractions of primaries heavier than protons as energy increases [4].

Above $\sim 5 \times 10^{19} \mathrm{eV}$ cosmic-ray protons interact with $\mathrm{CMB}$ photons and produce ultrahigh energy cosmogenic neutrinos of energies typically $1 / 20$ of the proton energy [7]. Their fluxes are uncertain and at EeV energies they depend mostly on the evolution with redshift $z$ of the unknown sources of UHECRs, and on their spectral features at injection. Protons typically produce more neutrinos than heavier primaries do [8,9], so measurement of the neutrino flux gives information on the nature of the primaries. In this respect the observation of UHE neutrinos can provide further hints on the dominant scenario of

\footnotetext{
${ }^{*}$ Deceased.

Now at Fermilab, Batavia, Illinois, USA.

${ }^{*}$ Now at CERN, Geneva, Switzerland.

${ }^{\S}$ Also at Vrije Universiteit Brussels, Belgium.

ๆauger_spokespersons@fnal.gov
}

Published by the American Physical Society under the terms of the Creative Commons Attribution 3.0 License. Further distribution of this work must maintain attribution to the author(s) and the published article's title, journal citation, and DOI.
UHECR production [9], as well as on the evolution with $z$ of their sources which can help in their identification $[9,10]$.

UHE neutrinos are also expected to be produced in the decay of charged pions created in the interactions of cosmic rays with matter and/or radiation at their potential sources, such as Gamma-Ray Bursts or Active Galactic Nuclei among others [11]. In fact, at tens of EeV, neutrinos may be the only direct probe of the sources of UHECRs at distances farther than $\sim 100 \mathrm{Mpc}$.

A breakthrough in the field was the recent detection with the IceCube experiment of three neutrinos of energies just above $1 \mathrm{PeV}$, including a $2 \mathrm{PeV}$ event which is the highestenergy neutrino interaction ever observed, followed by tens of others above $\sim 30 \mathrm{TeV}$ representing a $\sim 5.7 \sigma$ excess above atmospheric neutrino background [12]. The measured flux is close to the Waxman-Bahcall upper bound to the UHE neutrino flux [13], although with a steeper spectrum [14].

In the $\mathrm{EeV}$ energy range, i.e. about 3 orders of magnitude above the most energetic neutrinos detected in IceCube, neutrinos have so far escaped detection by existing experiments. These can be detected with a variety of techniques [15], among them with arrays of particle detectors at ground.

In this paper we report on the search for EeV neutrinos in data taken with the Surface Detector array (SD) of the Pierre Auger Observatory [16]. A blind scan of data from 1 January 2004 up to 20 June 2013 has yielded no neutrino candidates and an updated and stringent limit to the diffuse flux of UHE neutrino flux has been obtained.

\section{SEARCHING FOR UHE NEUTRINOS IN AUGER}

The concept for identification of neutrinos is rather simple. While protons, heavier nuclei, and even photons interact shortly after entering the atmosphere, neutrinos can initiate showers quite deep in the atmosphere. At large zenith angles the atmosphere is thick enough so that the electromagnetic component of nucleonic cosmic rays gets absorbed and the shower front at ground level is dominated 
by muons ("old" shower front). On the other hand, showers induced by neutrinos deep in the atmosphere have a considerable amount of electromagnetic component at the ground ("young" shower front). The Surface Detector array of the Pierre Auger Observatory is not directly sensitive to the muonic and electromagnetic components of the shower separately, nor to the depth at which the shower is initiated. In the 1600 waterCherenkov stations of the SD of the Pierre Auger Observatory, spread over an area of $\sim 3000 \mathrm{~km}^{2}$, separated by $1.5 \mathrm{~km}$ and arranged in a triangular grid, the signals produced by the passage of shower particles are digitized with flash analog to digital converters (FADC) with $25 \mathrm{~ns}$ resolution. This allows us to distinguish narrow signals in time induced by inclined showers initiated high in the atmosphere, from the broad signals expected in inclined showers initiated close to the ground.

Applying this simple idea, with the SD of the Pierre Auger Observatory [16] we can efficiently detect inclined showers and search for two types of neutrino-induced showers at energies above about $1 \mathrm{EeV}$ :

(1) Earth-skimming (ES) showers induced by tau neutrinos $\left(\nu_{\tau}\right)$ that travel in a slightly upward direction with respect to ground. $\nu_{\tau}$ can skim the Earth's crust and interact relatively close to the surface inducing a tau lepton which escapes the Earth and decays in flight in the atmosphere, close to the SD.

Typically, only Earth-skimming $\nu_{\tau}$-induced showers with zenith angles $90^{\circ}<\theta<95^{\circ}$ may be identified.

(2) Showers initiated by any neutrino flavor moving down at large angles with respect to the vertical that interact in the atmosphere close to the surface detector array through charged-current (CC) or neutral-current (NC) interactions. We include here showers induced by $\nu_{\tau}$ interacting in the mountains surrounding the Pierre Auger Observatory. Although this latter process is exactly equivalent to the "Earth-skimming" mechanism, it is included in this class because such showers are also going downwards. In the following we will refer to all these types of showers as "downward-going" (DG) $\nu$-induced showers.

With the aid of Monte Carlo simulations we have established that this search can be performed efficiently as long as it is restricted to showers with zenith angles $\theta>60^{\circ}$. Due to the characteristics of these showers depending on the zenith angle, the search in this channel was performed in two angular subranges: (a) "low" zenith angle (DGL) corresponding to $60^{\circ}<$ $\theta<75^{\circ}$ and (b) "high" zenith angle (DGH) with $75^{\circ}<\theta<90^{\circ}$.

\section{A. General procedure}

The identification of potential neutrino-induced showers is based on first selecting those events that arrive in rather inclined directions, and then selecting among them those with FADC traces that are spread in time, indicative of the early stage of development of the shower and a clear signature of a deeply interacting neutrino triggering the SD.

First of all, events occurring during periods of data acquisition instabilities [17] are excluded. For the remaining events the FADC traces of the triggered stations are first "cleaned" to remove accidental signals [18] induced mainly by random atmospheric muons arriving closely before or after the shower front. These muons are typically produced in lower energy showers (below the energy threshold of the $\mathrm{SD}$ of the Auger Observatory) that arrive by chance in coincidence with the triggering shower. A procedure to select the stations participating in the event described in $[18,19]$ is then applied, with the event accepted if the number of accepted stations $N_{\text {st }}$ is at least three (four) in the Earth-skimming (downward-going) selections.

From the pattern (footprint) of stations at ground a length $L$ along the arrival direction of the event and a width $W$ perpendicular to it characterizing the shape of the footprint are extracted [18]. The ratio $L / W \sim 1$ in vertical events, increasing gradually as the zenith angle increases. Very inclined events typically have elongated patterns on the ground along the direction of arrival and hence large values of $L / W$. A cut in $L / W$ is therefore a good discriminator of inclined events. Another indication of inclined events is given by the apparent speed $V$ of the trigger from a station $i$ to a station $j$, averaged over all pairs $(i, j)$ of stations in the event. This observable denoted as $\langle V\rangle$ is obtained from the distance between the stations after projection along $L$ and from the difference in trigger times of the stations. In vertical showers $\langle V\rangle$ exceeds the speed of light since all triggers occur at roughly the same time, while in very inclined events $\langle V\rangle$ is concentrated around the speed of light. Moreover its root-mean-square $[\mathrm{rms}(V)]$ value is small. For downward-going events only, a cut on the reconstructed zenith angle $\theta_{\text {rec }}$ is applied [19].

Once inclined showers are selected the next step is to identify young showers. A Time-over-Threshold (ToT) trigger $^{1}$ is usually present in SD stations with signals extended in time, while narrow signals induce other local triggers. Also the Area-over-Peak ratio (AoP), defined as the ratio of the integral of the FADC trace to its peak value, normalized to the average signal produced by a single muon, provides an estimate of the spread-in-time of the traces, and serves as an observable to discriminate broad from narrow shower fronts. In particular, a cut on AoP allows the rejection of background signals induced by inclined hadronic showers, in which the muons and their electromagnetic products

\footnotetext{
${ }^{1}$ This trigger is intended to select sequences of small signals in the FADC traces spread in time. It requires at least 13 bins in 120 FADC bins of a sliding window of $3 \mu$ s above a threshold of $0.2 I_{\mathrm{VEM}}^{\text {peak }}$ (the peak value of the signal expected for a vertical muon crossing the station), in coincidence in 2 out of 3 photomultiplier tubes (PMTs) [17].
} 
are concentrated within a short time interval, exhibiting AoP values close to the one measured in signals induced by isolated muons. These observables are used by themselves in the search for $\nu$ candidates, or combined in a linear Fisherdiscriminant polynomial depending on the selection as described later in this paper.

As a general procedure and to optimize the numerical values of the cuts and tune the algorithms needed to separate neutrino-induced showers from the much larger background of hadronic showers, we divided the whole data sample (1 January 2004-20 June 2013) into two parts (excluding periods of array instability). A selection dependent fraction of the data $20 \%$, along with Monte Carlo simulations of UHE neutrinos, is dedicated to define the selection algorithm, the most efficient observables and the value of the cuts on them. These data are assumed to be overwhelmingly constituted of background showers. The applied procedure is conservative because the presence of neutrinos in the training data would result in a more severe definition of the selection criteria. The remaining fraction of data is not used until the selection procedure is established, and then it is "unblinded" to search for neutrino candidates. We used real data to train the selections instead of Monte Carlo simulations of hadronic showers, the primary reason being that the detector simulation may not account for all possible detector fluctuations that may induce events that constitute a background to UHE neutrinos, while they are contained in data. It is important to remark that this is the same selection procedure and training period as in previous publications $[18,19]$, which is applied in this paper to a larger data set.

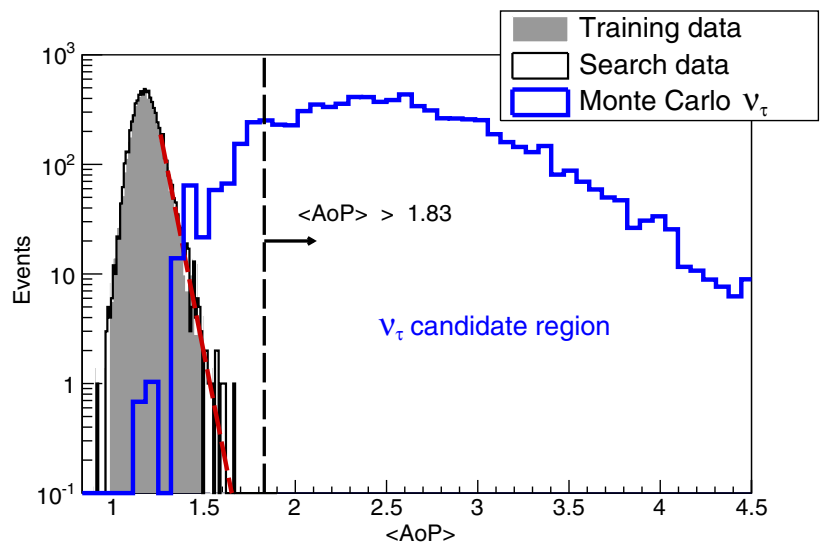

FIG. 1 (color online). Distributions of $\langle\mathrm{AoP}\rangle$ (the variable used to identifiy neutrinos in the ES selection for data after 1 June 2010) after applying the inclined shower selection in Table I. Gray-filled histogram: the data in the training period. Black histogram: data in the search period. These two distributions are normalized to the same number of events for comparison purposes. Blue histogram: simulated ES $\nu_{\tau}$ events. The dashed vertical line represents the cut on $\langle\mathrm{AoP}\rangle>1.83$ above which a data event is regarded as a neutrino candidate. An exponential fit to the tail of the distribution of training data is also shown as a red dashed line (see text for explanation).
Regarding the Monte Carlo simulations, the phase space of the neutrino showers reduces to three variables: the neutrino energy $E_{\nu}$, the incidence zenith angle $\theta$ and the interaction depth $D$ in the atmosphere for downward-going neutrinos, or the altitude $h_{c}$ of the $\tau$ decay above ground in the case of Earth-skimming neutrinos. Showers were simulated with energies from $\log \left(E_{\tau} / \mathrm{eV}\right)=17$ to 20.5 in steps of 0.5 , zenith angles from $90.1^{\circ}$ to $95.9^{\circ}$ in steps of $0.01 \mathrm{rad}(\mathrm{ES})$ and from $60^{\circ}$ to $90^{\circ}$ in steps of $0.05 \mathrm{rad}(\mathrm{DG})$. The values of $h_{c}$ range from 0 to $2500 \mathrm{~m}$ (in steps of $100 \mathrm{~m}$ ) whereas $D$ is uniformly distributed along the shower axis in steps of $100 \mathrm{~g} \mathrm{~cm}^{-2}$.

We have described the general procedure to search for Earth-skimming $\nu_{\tau}$ and downward-going $\nu$-induced showers. However the two searches (ES and DG) differ in several aspects that we describe in the following sections.

\section{B. Earth-skimming (ES) neutrinos}

With Monte Carlo simulations of UHE $\nu_{\tau}$ propagating inside the Earth, we have established that $\tau$ leptons above the energy threshold of the SD are efficiently produced only at zenith angles between $90^{\circ}$ and $95^{\circ}$. For this reason, in the Earth-skimming analysis we place very restrictive cuts to select only quasihorizontal showers with largely elongated footprints: $L / W>5$ and $\langle V\rangle \in[0.29,0.31] \mathrm{m} \mathrm{ns}^{-1}$ with $\operatorname{rms}(V)<0.08 \mathrm{mns}^{-1}$ (see Table I). ${ }^{2}$

In the ES selection, the neutrino identification variables include the fraction of stations with ToT trigger and having AoP > 1.4 for data prior to 31 May 2010 [18]. This fraction is required to be above $60 \%$ of the triggered stations in the event. The final choice of the values of these cuts was made by requiring zero background events in the training data sample, corresponding to $1 \%$ of the events recorded up to that date. For data beyond 1 June 2010 a new methodology and a new set of efficient selection criteria was established based on an improved and enlarged library of ES simulated $\nu_{\tau}$ events and on a larger period of training data. In particular, we used the average value of AoP ( $\langle$ AoP $\rangle$ ) over all the triggered stations in the event as the main observable to discriminate between hadronic showers and ES neutrinos. The new methodology allows us to place the value of the cut on $\langle\mathrm{AoP}\rangle$ using the tail of its distribution as obtained in real data (which was seen to be consistent with an exponential shape as shown in Fig. 1). This tail was fitted and extrapolated to find the value of the cut corresponding to less than 1 expected event per $50 \mathrm{yr}$ on the full SD array. As a result, an event is tagged as a neutrino candidate if $\langle\mathrm{AoP}\rangle>1.83$ (see Table I and Fig. 1). The new

\footnotetext{
${ }^{2}$ The axis of Earth-skimming showers traveling in the upward direction does not intersect the ground, contrary to the case for downward-going showers. For this reason, we exploit the properties of the footprint generated by the shower particles that deviate laterally from the shower axis and trigger the SD waterCherenkov stations.
} 
methodology is not applied to the data prior to 31 May 2010 since that data period was already unblinded to search for UHE neutrinos under the older cuts [18].

Roughly $\sim 95 \%$ of the simulated inclined $\nu_{\tau}$ events producing $\tau$ leptons above the energy threshold of the $\mathrm{SD}$ are kept after the cut on $\langle\mathrm{AoP}\rangle$. The search for neutrinos is clearly not limited by background in this channel.

\section{Downward-going (DG) neutrinos}

In the high zenith angle range of the downward-going analysis (DGH) the values of the cuts to select inclined events are obtained in Monte Carlo simulations of events with $\theta>75^{\circ}$. Due to the larger angular range compared to Earth-skimming $\nu_{\tau}$, less stringent criteria are applied, namely $L / W>3,\langle V\rangle<0.313 \mathrm{~m} \mathrm{~ns}^{-1}, \operatorname{rms}(V) /\langle V\rangle<0.08$ plus a further requirement that the reconstructed zenith angle $\theta_{\text {rec }}>75^{\circ}$ (see [19] and Table I for full details).

In the low zenith angle range (DGL) corresponding to $60^{\circ}<\theta<75^{\circ}, L / W,\langle V\rangle$ and $\operatorname{rms}(V) /\langle V\rangle$ are less efficient in selecting inclined events than the reconstructed zenith angle $\theta_{\text {rec }}$, and for this reason only a cut on $\theta_{\text {rec }}$ is applied, namely $58.5^{\circ}<\theta_{\text {rec }}<76.5^{\circ}$, which includes some allowance to account for the resolution in the angular reconstruction of the simulated neutrino events.

After the inclined shower selection is performed, the discrimination power is optimized with the aid of the multivariate Fisher discriminant method [20]. A linear combination of observables is constructed which optimizes the separation between background hadronic inclined showers occurring during the downward-going training period, and Monte Carlo simulated $\nu$-induced showers. The method requires as input a set of observables. For that purpose we use variables depending on the dimensionless Area-over-Peak (AoP) observable_ as defined above—of the FADC traces.
In the DGH channel, due to the inclination of the shower the electromagnetic component is less attenuated at the locations of the stations that are first hit by a deep inclined shower (early stations) than in the stations that are hit last (late stations). From Monte Carlo simulations of $\nu$-induced showers with $\theta>75^{\circ}$ we have established that in the first few early stations the typical AoP values range between 3 and 5, while AoP tends to be closer to 1 in the late stations. Based on this simple observation and as already reported in [19], we have found a good discrimination when the following ten variables are used to construct the linear Fisher discriminant variable $\mathcal{F}$ : the $\mathrm{AoP}$ and $(\mathrm{AoP})^{2}$ of the four stations that trigger first in each event, the product of the four AoPs, and a global parameter that measures the asymmetry between the average AoP of the early stations and those triggering last in the event (see [19] for further details and Table I).

The selection of neutrino candidates in the zenith angle range $60^{\circ}<\theta<75^{\circ}$ (DGL) is more challenging since the electromagnetic component of background hadronic showers at ground increases as the zenith angle decreases because the shower crosses less atmosphere before reaching the detector level. Out of all triggered stations of an event in this angular range, the ones closest to the shower core exhibit the highest discrimination power in terms of AoP. In fact it has been observed in Monte Carlo simulations that the first triggered stations can still contain some electromagnetic component for background events and, for this reason, it is not desirable to use them for discrimination purposes. The last ones, even if they are triggered only by muons from a background hadronic shower, can exhibit large values of AoP because they are far from the core where muons are known to arrive with a larger spread in time. Based on the information from Monte Carlo simulations, the variables used in the Fisher discriminant

TABLE I. Observables and numerical values of cuts applied to select inclined and young showers for Earth-skimming and downwardgoing neutrinos. See text for explanation.

\begin{tabular}{|c|c|c|c|}
\hline Selection & Earth-skimming (ES) & $\begin{array}{l}\text { Downward-going } \\
\text { high angle (DGH) }\end{array}$ & $\begin{array}{l}\text { Downward-going } \\
\text { low angle (DGL) }\end{array}$ \\
\hline $\begin{array}{l}\text { Flavours and interactions } \\
\text { Angular range } \\
\mathrm{N}^{\circ} \text { of stations }\left(N_{\mathrm{st}}\right)\end{array}$ & $\begin{array}{l}\nu_{\tau} \mathrm{CC} \\
\theta>90^{\circ} \\
N_{\mathrm{st}} \geq 3\end{array}$ & $\begin{array}{c}\nu_{e}, \nu_{\mu}, \nu_{\tau} \mathrm{CC} \& \mathrm{NC} \\
\theta \in\left(75^{\circ}, 90^{\circ}\right) \\
N_{\mathrm{st}} \geq 4\end{array}$ & $\begin{array}{c}\nu_{e}, \nu_{\mu}, \nu_{\tau} \mathrm{CC} \& \mathrm{NC} \\
\theta \in\left(60^{\circ}, 75^{\circ}\right) \\
N_{\mathrm{st}} \geq 4\end{array}$ \\
\hline Inclined showers & $\begin{array}{c}L / W>5 \\
\langle V\rangle \in(0.29,0.31) \mathrm{m} \mathrm{ns}^{-1} \\
\operatorname{rms}(V)<0.08 \mathrm{~m} \mathrm{~ns}^{-1}\end{array}$ & $\begin{array}{c}\theta_{\text {rec }}>75^{\circ} \\
L / W>3 \\
\langle V\rangle<0.313 \mathrm{mns}^{-1} \\
\operatorname{rms}(V) /\langle V\rangle<0.08\end{array}$ & $\theta_{\mathrm{rec}} \in\left(58.5^{\circ}, 76.5^{\circ}\right)$ \\
\hline Young showers & $\begin{array}{c}\text { Data: } 1 \text { January } 2004-31 \text { May } 2010 \\
\geq 60 \% \text { of stations with } \\
\text { ToT trigger and AoP }>1.4 \\
\text { Data: } 1 \text { June } 2010-20 \text { June } 2013 \\
\quad\langle\text { AoP }\rangle>1.83 \\
\mathrm{AoP}_{\min }>1.4 \text { if } N_{\mathrm{st}}=3\end{array}$ & $\begin{array}{l}\text { Fisher discriminant based } \\
\text { on AoP of early stations }\end{array}$ & $\begin{array}{l}\geq 75 \% \text { of stations close to } \\
\text { shower core with ToT trigger } \\
\text { and } \\
\text { Fisher discriminant based } \\
\text { on AoP of early stations } \\
\text { close to shower core }\end{array}$ \\
\hline
\end{tabular}




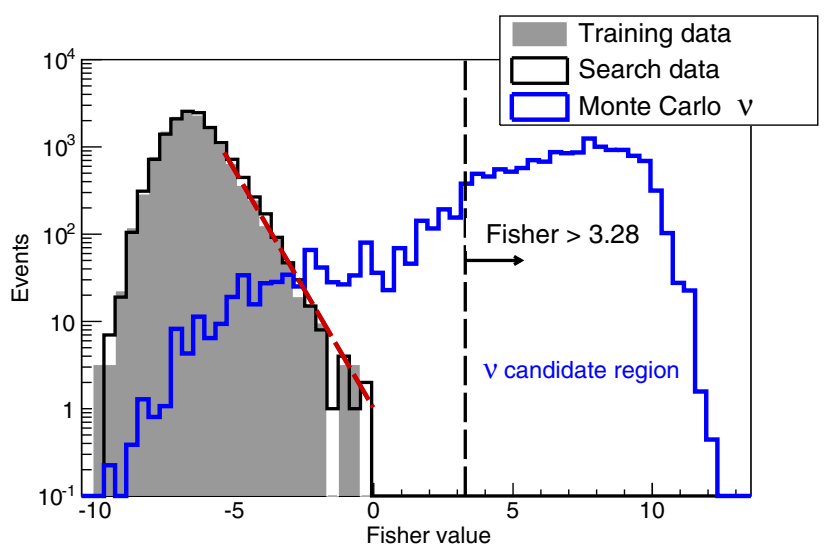

FIG. 2 (color online). Distributions of the Fisher variable $\mathcal{F}$ in inclined events selected by the "Inclined showers" DGH criteria in Table I, before applying the "Young showers" cuts. In particular the distribution of events with number of triggered tanks $7 \leq N_{\text {st }} \leq 11$ is shown. Gray-filled histogram: data in the training period corresponding to $\sim 23 \%$ of the whole data sample between 1 January 2004 and 20 June 2013. Black line: data in the search period. The distributions are normalized to the same number of events for comparison purposes. Blue line: simulated DGH $\nu$ events. The dashed vertical line represents the cut on $\mathcal{F}>3.28$ above which a data event is regarded as a neutrino candidate. The red dashed line represents an exponential fit to the tail of the training distribution (see text for explanation).

analysis are the individual AoP of the four or five stations (depending on the zenith angle) closest to the core, and their product [21]. In the DGL analysis it is also required that at least $75 \%$ of the triggered stations closest to the core have a ToT local trigger [21].

Once the Fisher discriminant $\mathcal{F}$ is defined, the next step is to define a numerical value $\mathcal{F}_{\text {cut }}$ that efficiently separates neutrino candidates from regular hadronic showers. As was done for the variable $\langle\mathrm{AoP}\rangle$ in the Earth-skimming analysis, $\mathcal{F}_{\text {cut }}$ was fixed using the tail of the distribution of $\mathcal{F}$ in real data, which is consistent with an exponential shape in all cases. An example is shown in Fig. 2. The tail was fitted and extrapolated to find the value of $\mathcal{F}_{\text {cut }}$ corresponding to less than 1 expected event per $50 \mathrm{yr}$ on the full SD array $[19,21]$. Roughly $\sim 85 \%$ ( $60 \%)$ of the simulated inclined $\nu$ events are kept after the cut on the Fisher variable in the DGH (DGL) selections. The smaller efficiencies for the identification of neutrinos in the DGL selection are due to the more stringent criteria in the angular bin $\theta \in\left(60^{\circ}, 75^{\circ}\right)$ needed to reject the larger contamination from cosmic-ray induced showers.

\section{DATA UNBLINDING AND EXPOSURE CALCULATION}

\section{A. Data unblinding}

No events survived when the Earth-skimming and downward-going selection criteria explained above and summarized in Table I are applied blindly to the data collected between 1 January 2004 and 20 June 2013. For each selection the corresponding training periods are excluded from the search. After the unblinding we tested the compatibility of the distributions of discriminating observables in the search and training samples. Examples are shown in Fig. 1 for the $\langle\mathrm{AoP}\rangle$ variable in the Earth-skimming analysis, and in Fig. 2 for the Fisher variable in the DGH analysis. In particular fitting the tails of the corresponding distributions to an exponential, we obtained compatible parameters within $1 \sigma$ statistical uncertainties.

\section{B. Exposure calculation}

\section{Neutrino identification efficiencies}

The selection criteria in Table I, were also applied to neutrino-induced showers simulated with Monte Carlo, and the identification efficiencies $\epsilon_{\mathrm{ES}}, \epsilon_{\mathrm{DGH}}, \epsilon_{\mathrm{DGL}}$ for each channel-defined as the fraction of simulated events passing the cuts-were obtained.

A large set of Monte Carlo simulations of neutrinoinduced showers was performed for this purpose, covering the whole parameter space where the efficiency is expected to be sizable. In the case of Earth-skimming $\nu_{\tau}$ induced showers, the efficiency depends on the energy of the emerging $\tau$ leptons $E_{\tau}$, on the zenith angle $\theta$ and on the altitude of the decay point of the $\tau$ above ground. These efficiencies are averaged over azimuthal angle and the $\tau$ decay channels. The maximum efficiency that can be reached is $82.6 \%$, the $17.4 \%$ remaining corresponds to the channel in which the $\tau$ decays into a $\mu$ which is unlikely to produce a detectable shower close to ground. In the case of downward-going neutrinos the identification efficiency depends on neutrino flavor, type of interaction (CC or NC), neutrino energy $E_{\nu}$, zenith angle $\theta$, and distance $D$ measured from ground along the shower axis at which the neutrino is forced to interact in the simulations.

The identification efficiencies depend also on time, through the changing configuration of the SD array that was growing steadily since 2004 up to 2008, and because the fraction of working stations-although typically above 95\% - is changing continuously with time. Also the continuous monitoring of the array reveals a slight evolution with time of the optical properties of the water-Cherenkov stations (see below). Although the number of working stations and their status are monitored every second and as a consequence the SD configuration is known with very good accuracy at any instant of time, in practice, to avoid having to cope with an impractically large number of configurations, different strategies were devised to calculate in an accurate and less time-consuming manner the actual identification efficiencies (as explained in $[18,19,21])$. 
The evolution of the optical properties of the waterCherenkov stations was taken into account in an effective way in the calculation of the exposure. The main effect of this evolution is a decrease with time of the decay time of the light as obtained from the monitoring data that revealed a continuous decrease of $\sim 10 \%$ from 2004 until the end of the data period used in this work (20 June 2013). This induces a reduction of the AoP and, as a consequence, the trigger efficiency changes with time. These changes were accounted for in the calculation of the exposure by dividing the whole data set into three separate periods and assuming that in each of them the decay time of the light in the tank remained approximately constant as seen in data. A conservative approach was adopted by choosing constant values of the light decay time below the actual curve in the three periods.

\section{Combination of selections}

In previous publications [18,19,21] the fraction of $\nu$-induced Monte Carlo events identified as neutrino candidates was obtained by applying each particular set of selection criteria (ES, DGH, DGL) only to its corresponding set of simulated showers (ES, DGH or DGL). In this paper the fraction of selected events is further increased by applying the three sets of criteria to each sample of simulated showers (ES, DGH, DGL) regardless of channel. With this procedure the fraction of identified Monte Carlo events is enhanced as, for instance, an ES simulated shower induced by a $\nu_{\tau}$ might not fulfill the requirements of the ES selection, but might still pass the DGH or DGL criteria, and hence contribute to the fraction of identified events. The enhancement in the fraction of events when applying this "combined" analysis depends on the particular set of Monte Carlo simulations. For instance applying the three criteria to the DGH Monte Carlo sample identifies a fraction of neutrino events $\sim 1.25$ larger than when the DGH criteria are applied alone, the enhancement coming mainly from events with three stations rejected by the DGH criteria but accepted by ES. The application of the three criteria to the ES Monte Carlo sample however results in a smaller enhancement $\sim 1.04$.

\section{Exposure calculation}

For downward-going neutrinos, once the efficiencies $\epsilon_{\mathrm{DG}}\left(E_{\nu}, \theta, D, t\right)$ are obtained, the calculation of the exposure involves folding them with the SD array aperture and the $\nu$ interaction probability at a depth $D$ for a neutrino energy $E_{\nu}$. This calculation also includes the possibility that downward-going $\nu_{\tau}$ interact with the mountains surrounding the Observatory. Integrating over the parameter space except for $E_{\nu}$ and in time over the search periods and summing over all the interaction channels yields the exposure [19,21].

In the Earth-skimming channel, $\epsilon_{\mathrm{ES}}\left(E_{\tau}, \theta, X_{d}\right)$ are also folded with the aperture, with the probability density function of a tau emerging from the Earth with energy $E_{\tau}$ (given a neutrino with energy $E_{\nu}$ crossing an amount of Earth determined by the zenith angle $\theta$ ), as well as with the probability that the $\tau$ decays at an altitude $h_{c}$ [18]. An integration over the whole parameter space except for $E_{\nu}$ and time gives the exposure [18].

The exposures $\mathcal{E}_{\mathrm{ES}}, \mathcal{E}_{\mathrm{DGH}}$ and $\mathcal{E}_{\mathrm{DGL}}$ obtained for the search periods of each selection are plotted in Fig. 3 along with their sum $\mathcal{E}_{\text {tot }}$. The exposure to Earth-skimming neutrinos is higher than that to downward-going neutrinos, partially due to the longer search period in the Earthskimming analysis, and partially due to the much larger neutrino conversion probability in the denser target of the Earth's crust compared to the atmosphere. The larger number of neutrino flavors and interaction channels that can be identified in the DGH and DGL analysis, as well as the broader angular range $60^{\circ}<\theta<90^{\circ}$ partly compensates the dominance of the ES channel. The ES exposure flattens and then falls above $\sim 10^{19} \mathrm{eV}$ as there is an increasing probability that the $\tau$ decays high in the atmosphere producing a shower not triggering the array, or even that the $\tau$ escapes the atmosphere before decaying. At the highest energies the DGH exposure dominates. The DGL exposure is the smallest of the three, mainly due to the more stringent criteria needed to apply to get rid of the larger background nucleonic showers in the zenith angle bin $60^{\circ}<\theta<75^{\circ}$.

The relative contributions of the three channels to the total expected event rate for a differential flux behaving with energy as $d N_{\nu}\left(E_{\nu}\right) / d E_{\nu} \propto E_{\nu}^{-2}$ are $\mathrm{ES}: \mathrm{DGH}: \mathrm{DGL} \sim 0.84: 0.14: 0.02$ respectively, where the event rate is obtained as

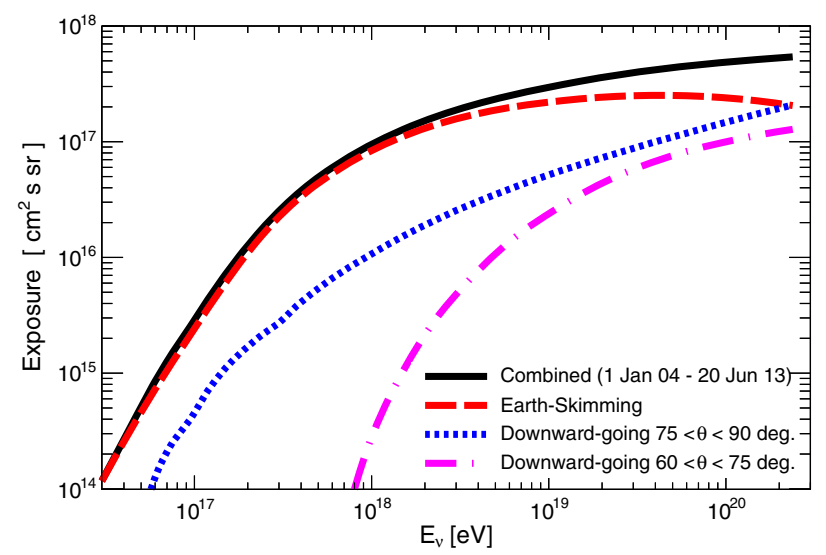

FIG. 3 (color online). Combined exposure of the SD of the Pierre Auger Observatory (1 January 2004-20 June 2013) as a function of neutrino energy after applying the three sets of selection criteria in Table I to Monte Carlo simulations of UHE neutrinos (see text for explanation). Also shown are the individual exposures corresponding to each of the three selections. For the downward-going channels the exposure represents the sum over the three neutrino flavors as well as $\mathrm{CC}$ and NC interactions. For the Earth-skimming channel, only $\nu_{\tau} \mathrm{CC}$ interactions are relevant. 


$$
N_{\mathrm{evt}}=\int_{E_{\nu}} \frac{d N_{\nu}}{d E_{\nu}}\left(E_{\nu}\right) \mathcal{E}_{\mathrm{tot}}\left(E_{\nu}\right) d E_{\nu} .
$$

\section{Systematic uncertainties}

Several sources of systematic uncertainty have been considered. Some of them are directly related to the Monte Carlo simulation of the showers, i.e., generator of the neutrino interaction either in the Earth or in the atmosphere, parton distribution function, air shower development, and hadronic model.

Other uncertainties have to do with the limitations on the theoretical models needed to obtain the interaction cross section or the $\tau$ energy loss at high energies. In the Earthskimming analysis the model of energy loss for the $\tau$ is the dominant source of uncertainty, since it determines the energy of the emerging $\tau$ s after propagation in the Earth; the impact of this on the downward-going analysis is much smaller since $\tau$ energy losses are only relevant for $\nu_{\tau}$ interacting in the mountains, a channel that is estimated to contribute only $\sim 15 \%$ to the DGH exposure [19].

The uncertainty on the shower simulation, which stems mainly from the different shower propagation codes and hadronic interaction models that can be used to model the high energy collisions in the shower, contributes significantly in the ES and DG channels.

The presence of mountains around the Observatorywhich would increase the target for neutrino interactions in both cases - is explicitly simulated and accounted for when obtaining the exposure of the SD to downward-going neutrino-induced showers, and as a consequence does not contribute directly to the systematic uncertainties. However, it is not accounted for in the Earth-skimming channel and instead we take the topography around the Observatory as a source of systematic uncertainty.

In the three channels the procedure to incorporate the systematic uncertainties is the same. Different combinations of the various sources of systematic uncertainty render different values of the exposure and a systematic uncertainty band of relative deviation from a reference exposure (see below) can be constructed for each channel and for each source of systematic uncertainty. For a given source of uncertainty the edges of the ES, DGH and DGL bands are weighted by the relative importance of each channel as given before and added linearly or quadratically depending on the source of uncertainty. In Table II we give the dominant sources of systematic uncertainty and their corresponding combined uncertainty bands obtained in this way. The combined uncertainty band is then incorporated in the value of the limit itself through a semi-Bayesian extension [22] of the Feldman-Cousins approach [23].

In the calculation of the reference exposure the $\nu$-nucleon interaction in the atmosphere for DG neutrinos (including CC and NC channels) is simulated with HERWIG [24].
TABLE II. Main sources of systematic uncertainties and their corresponding combined uncertainty bands (see text for details) representing the effect on the event rate defined in Eq. (1). The uncertainty due to "Simulations" includes: interaction generator, shower simulation, hadronic model, thinning and detector simulator. The uncertainty due to " $\tau$ energy-loss" affects the ES channel and also the DGH but only to $\nu_{\tau}$ with $\theta \gtrsim 88^{\circ}$ going through the mountains surrounding the Pierre Auger Observatory. However it does not affect the DGL channel. The topography around the Observatory is not accounted for in the ES channel and is taken as a systematic uncertainty that would increase the event rate.

\begin{tabular}{lc}
\hline \hline Source of systematic & Combined uncertainty band \\
\hline Simulations & $\sim+4 \%,-3 \%$ \\
$\nu$ cross section and $\tau$ E-loss & $\sim+34 \%,-28 \%$ \\
Topography & $\sim+15 \%, 0 \%$ \\
Total & $\sim+37 \%,-28 \%$ \\
\hline \hline
\end{tabular}

In the case of $\nu_{\tau} \mathrm{CC}$ interactions, a dedicated, fast and flexible code is used to simulate the $\tau$ lepton propagation in the Earth and/or in the atmosphere. The $\tau$ decay is performed with the TAUOLA package [25]. In all cases we adopted the $\nu$-nucleon cross section in [26]. In a second step, the AIRES code [27] is used to simulate the propagation in the atmosphere of the particles produced in the high energy $\nu$ interaction or in the $\tau$ lepton decay. The types, energies, momenta and times of the particles reaching the SD level are obtained. The last stage is the simulation of the SD response (PMT signals and FADC traces). This involves a modification of the "standard" sampling procedure in [28] to regenerate particles in the SD stations from the "thinned" air shower simulation output, which was tailored to the highly inclined showers involved in the search for neutrinos. Light production and propagation inside the station is based on GEANT4 [29] with the modifications to account for the evolution of the light decay time explained above. These two latter changes roughly compensate each other, with the net result being a few percent decrease of the exposure with respect to that obtained with the standard thinning procedure and a constant average value of the light decay-time.

\section{RESULTS}

Using the combined exposure in Fig. 3 and assuming a differential neutrino flux $d N\left(E_{\nu}\right) / d E_{\nu}=k \cdot E_{\nu}^{-2}$ as well as a $\nu_{e}: \nu_{\mu}: \nu_{\tau}=1: 1: 1$ flavor ratio, an upper limit on the value of $k$ can be obtained as

$$
k=\frac{N_{\mathrm{up}}}{\int_{E_{\nu}} E_{\nu}^{-2} \mathcal{E}_{\mathrm{tot}}\left(E_{\nu}\right) d E_{\nu}}
$$

The actual value of the upper limit on the signal events $\left(N_{\text {up }}\right)$ depends on the number of observed events ( 0 in our 


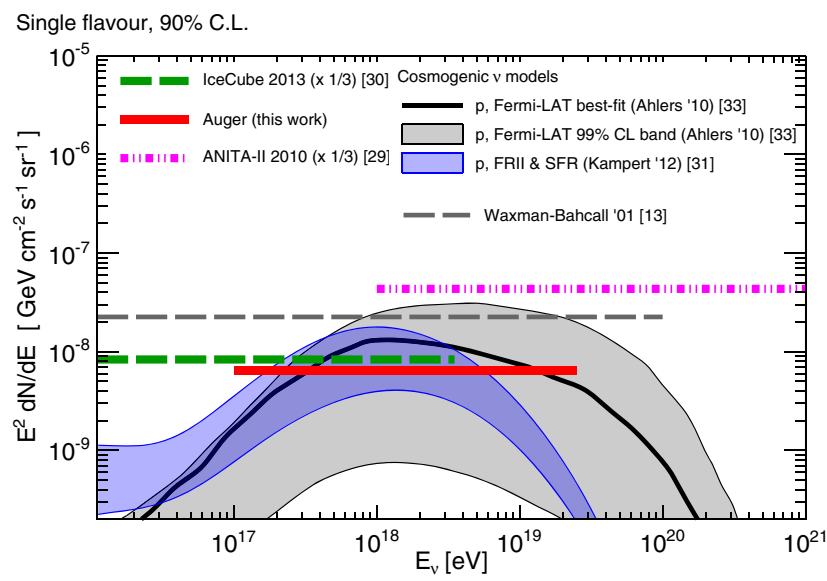

Single flavour, $90 \%$ C.L.

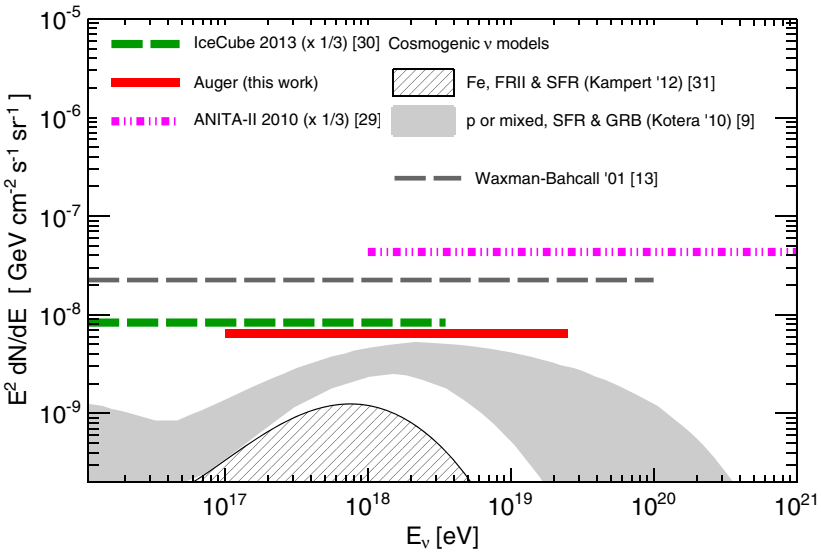

FIG. 4 (color online). Top panel: Upper limit (at 90\% C.L.) to the normalization of the diffuse flux of UHE neutrinos as given in Eqs. (2) and (3), from the Pierre Auger Observatory. We also show the corresponding limits from ANITAII [31] and IceCube [32] experiments, along with expected fluxes for several cosmogenic neutrino models that assume pure protons as primaries $[33,34]$ as well as the Waxman-Bahcall bound [13]. All limits and fluxes converted to single flavor. We used $N_{\text {up }}=2.39$ in Eq. (2) to obtain the limit (see text for details). Bottom panel: Same as top panel, but showing several cosmogenic neutrino models that assume heavier nuclei as primaries, either pure iron [33] or mixed primary compositions [9].

case) and expected background events (conservatively assumed to be 0), as well as on the confidence level required (90\% C.L. in the following). Using a semiBayesian extension [22] of the Feldman-Cousins approach [23] to include the uncertainties in the exposure we obtain ${ }^{3}$ $N_{\text {up }}=2.39$. The single-flavor $90 \%$ C.L. limit is

$$
k_{90}<6.4 \times 10^{-9} \mathrm{GeV} \mathrm{cm}^{-2} \mathrm{~s}^{-1} \mathrm{sr}^{-1} .
$$

\footnotetext{
${ }^{3}$ To calculate $N_{\text {up }}$ we use POLE ++ [22]. The signal efficiency uncertainty is $\sim 0.19$ with an asymmetric band (see Table II). This yields a value of $N_{\text {up }}=2.39$ slightly smaller than the nominal 2.44 of the Feldman-Cousins approach.
}

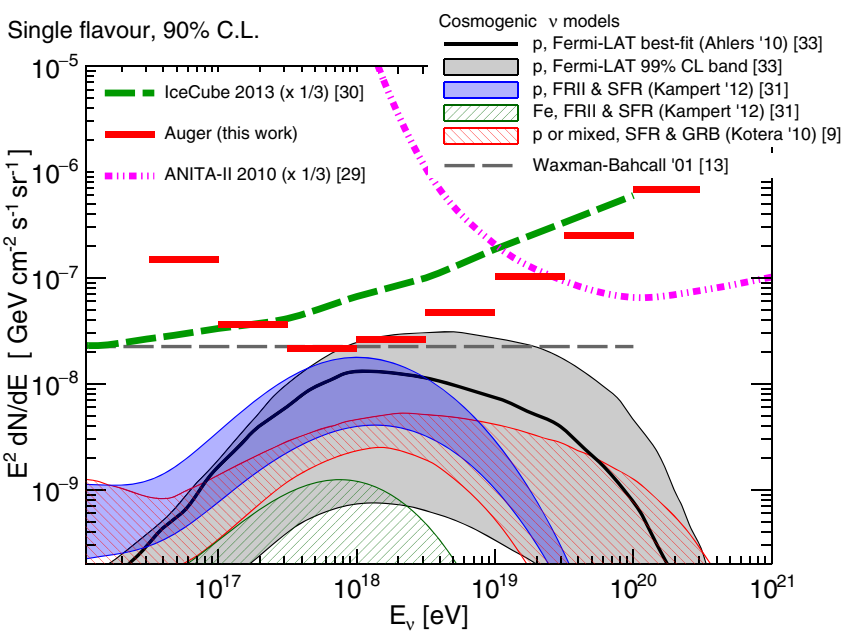

FIG. 5 (color online). Upper limit to the normalization of the diffuse flux of UHE neutrinos (at 90\% C.L. and in bins of width 0.5 in $\log _{10} E_{\nu}$ - see text for details) from the Pierre Auger Observatory (straight steps). We also show the corresponding limits from ANITAII [31] (dot-dashed line) and IceCube [32] (dashed line) experiments (with appropriate normalizations to take into account the energy bin width, and to convert to single flavor), along with expected fluxes for several cosmogenic neutrino models $[9,33,34]$ as well as the Waxman-Bahcall bound [13] (all converted to single flavor).

The limit applies in the energy interval $\sim 1.0 \times$ $10^{17} \mathrm{eV}-2.5 \times 10^{19} \mathrm{eV}$ where the cumulative number of events as a function of neutrino energy increases from $5 \%$ to $95 \%$ of the total number, i.e. where $\sim 90 \%$ of the total event rate is expected. It is important to remark that this is the most stringent limit obtained so far with Auger data, and it represents a single limit combining the three channels where we have searched for UHE neutrinos. The limit to the flux normalization in Eq. (3) is obtained integrating the denominator of Eq. (2) in the whole energy range where Auger is sensitive to UHE neutrinos. This is shown in Fig. 4, along with the $90 \%$ C.L. limits from other experiments as well as several models of neutrino flux production (see caption for references). The denominator of Eq. (2) can also be integrated in bins of energy, and a limit on $k$ can also be obtained in each energy bin [30]. This is displayed in Fig. 5 where the energy bins have a width of 0.5 in $\log _{10} E_{\nu}$, and where we also show the whole energy range where there is sensitivity to neutrinos. The limit as displayed in Fig. 5 allows us to show at which energies the sensitivity of the SD of the Pierre Auger Observatory peaks.

The search period corresponds to an equivalent of 6.4 years of a complete Auger SD array working continuously. The inclusion of the data from 1 June 2010 until 20 June 2013 in the search represents an increase of a factor $\sim 1.8$ in total time quantified in terms of equivalent full Auger years with respect to previous searches $[18,19]$. Further improvements in the limit come from the combination of the three 
TABLE III. Number of expected events $N_{\text {evt }}$ in Eq. (1) for several theoretical models of UHE neutrino production (see Figs. 4 and 5), given the combined exposure of the surface detector array of the Pierre Auger Observatory plotted in Fig. 3. The last column gives the Poisson probability $\exp \left(-N_{\text {evt }}\right)$ of observing 0 events when the number of expected events is $N_{\text {evt }}$ given in the second column.

\begin{tabular}{lcc}
\hline \hline Diffuse flux Neutrino model & $\begin{array}{c}\text { Expected number of events } \\
\text { (1 January 2004-20 June 2013) }\end{array}$ & $\begin{array}{c}\text { Probability of } \\
\text { observing 0 }\end{array}$ \\
\hline Cosmogenic_proton, FRII [33] & $\sim 4.0$ & $\sim 1.8 \times 10^{-2}$ \\
Cosmogenic-proton, SFR [33] & $\sim 0.9$ & $\sim 0.4$ \\
Cosmogenic-proton, Fermi-LAT, $E_{\min }=10^{19} \mathrm{eV} \mathrm{[34]}$ & $\sim 3.2$ & $\sim 4 \times 10^{-2}$ \\
Cosmogenic-proton, Fermi-LAT, $E_{\min }=10^{17.5} \mathrm{eV} \mathrm{[34]}$ & $\sim 1.6$ & $\sim 0.2$ \\
Cosmogenic-proton or mixed, SFR \& GRB [9] & $\sim 0.5-1.4$ & $\sim 0.6-0.2$ \\
Cosmogenic_iron, FRII [33] & $\sim 0.3$ & $\sim 0.7$ \\
Astrophysical $\nu$ (AGN) [35] & $\sim 7.2$ & $\sim 7 \times 10^{-4}$ \\
Exotic [36] & $\sim 31.5$ & $\sim 2 \times 10^{-14}$ \\
\hline \hline
\end{tabular}

analyses into a single one, using the procedure explained before that enhances the fraction of identified neutrinos especially in the DGH channel.

In Table III we give the expected total event rates for several models of neutrino flux production.

Several important conclusions and remarks can be stated after inspecting Figs. 4 and 5 and Table III:

(1) The maximum sensitivity of the SD of the Auger Observatory is achieved at neutrino energies around $\mathrm{EeV}$, where most cosmogenic models of $\nu$ production also peak (in a $E_{\nu}^{2} \times d N / d E_{\nu}$ plot).

(2) The current Auger limit is a factor $\sim 4$ below the Waxman-Bahcall bound on neutrino production in optically thin sources [13]. The SD of the Auger Observatory is the first air shower array to reach that level of sensitivity.

(3) Some models of neutrino production in astrophysical sources such as Active Galactic Nuclei (AGN) are excluded at more than $90 \%$ C.L. For the model \#2 shown in Fig. 14 of [35] we expect $\sim 7$ neutrino events while none was observed.

(4) Cosmogenic $\nu$ models that assume a pure primary proton composition injected at the sources and strong (FRII-type) evolution of the sources are strongly disfavored by Auger data. An example is the upper line of the shaded band in Fig. 17 in [33] (also depicted in Figs. 4 and 5), for which $\sim 4$ events are expected and as a consequence that flux is excluded at $\sim 98 \%$ C.L. Models that assume a pure primary proton composition and use the $\mathrm{GeV} \gamma$-ray flux observations by the Fermi-LAT satellite detector as an additional constraint, are also disfavored. For instance for the model shown as a solid line in the bottom right panel of Fig. 5 in [34] (also depicted in Figs. 4 and 5 in this work), corresponding to the bestfit to the cosmic-ray spectrum as measured by HiRes, we expect $\sim 3.2$ events. As a consequence that model is excluded at more than $90 \%$ C.L. For this particular model we also show in Figs. 4 and 5 the 99\% C.L. band resulting from the fitting to the HiRes spectrum down to $E_{\min }=10^{19} \mathrm{eV}$. The Auger limit is also approaching the solid line in the upper left panel of Fig. 5 in [34], a model that assumes extragalactic protons above $E_{\min }=10^{17.5} \mathrm{eV}$ [37], for which $\sim 1.6$ events are expected (see Table III). The Auger direct limits on cosmogenic neutrinos are also constraining part of the region indirectly bounded by Fermi-LAT observations.

(5) The current Auger limit is less restrictive with the cosmogenic neutrino models represented by the gray shaded area in the bottom panel of Fig. 4 ( $\sim 0.5$ to $\sim 1.4$ events are expected as shown in Table III) which brackets the lower fluxes predicted under a range of assumptions for the composition of the primary flux (protons or mixed), source evolution and model for the transition from Galactic to extragalactic cosmic rays [9] The same remark applies to models that assume pure-iron composition at the sources. A tenfold increase in the current exposure will be needed to reach the most optimistic predictions of cosmogenic neutrino fluxes if the primaries are pure iron, clearly out of the range of the current configuration of the Auger Observatory.

(6) A large range of exotic models of neutrino production [36] are excluded with C.L. larger than $99 \%$.

(7) In IceCube, neutrino fluxes in the $30 \mathrm{TeV}$ to $2 \mathrm{PeV}$ energy range have shown a $\sim 5.7 \sigma$ excess compared to predicted atmospheric neutrino fluxes [12]. A refinement of the IceCube search technique to extend the neutrino sensitivity down to $10 \mathrm{TeV}$ [14] yielded a power-law fit to the measured flux without cutoff given by $d N / d E=\Phi_{0}\left(E_{\nu} / E_{0}\right)^{-\gamma}$ with $\Phi_{0}=2.06 \times 10^{-18} \mathrm{GeV}^{-1} \mathrm{~cm}^{-2} \mathrm{~s}^{-1} \mathrm{sr}^{-1}, E_{0}=$ $10^{5} \mathrm{GeV}$, and $\gamma=2.46$. If this flux is extrapolated to $10^{20} \mathrm{eV}$ it would produce $\sim 0.1$ events in Auger.

\section{ACKNOWLEDGMENTS}

The successful installation, commissioning, and operation of the Pierre Auger Observatory would not have been possible without the strong commitment and effort from the 
technical and administrative staff in Malargüe. We are very grateful to the following agencies and organizations for financial support: Comisión Nacional de Energía Atómica, Fundación Antorchas, Gobierno de la Provincia de Mendoza, Municipalidad de Malargüe, NDM Holdings and Valle Las Leñas, in gratitude for their continuing cooperation over land access, Argentina; the Australian Research Council; Conselho Nacional de Desenvolvimento Científico e Tecnológico (CNPq), Financiadora de Estudos e Projetos (FINEP), Fundação de Amparo à Pesquisa do Estado de Rio de Janeiro (FAPERJ), São Paulo Research Foundation (FAPESP) Grants No. 2010/07359-6 and No. 1999/05404-3, Ministério de Ciência e Tecnologia (MCT), Brazil; Grants No. MSMT-CR LG13007, No. 7AMB14AR005, and the Czech Science Foundation Grant No. 14-17501S, Czech Republic; Centre de Calcul IN2P3/CNRS, Centre National de la Recherche Scientifique (CNRS), Conseil Régional Ile-de-France, Département Physique Nucléaire et Corpusculaire (PNC-IN2P3/CNRS), Département Sciences de l'Univers (SDU-INSU/CNRS), Institut Lagrange de Paris (ILP) Grant No. LABEX ANR-10-LABX-63, within the Investissements d'Avenir Programme Grant No. ANR11-IDEX-0004-02, France; Bundesministerium für Bildung und Forschung (BMBF), Deutsche Forschungsgemeinschaft (DFG), Finanzministerium Baden-Württemberg, Helmholtz Alliance for Astroparticle Physics (HAP), Helmholtz-Gemeinschaft Deutscher Forschungszentren (HGF), Ministerium für Wissenschaft und Forschung, Nordrhein Westfalen, Ministerium für Wissenschaft, Forschung und Kunst, Baden-Württemberg, Germany; Istituto Nazionale di Fisica Nucleare (INFN), Ministero dell'Istruzione, dell'Universitá e della Ricerca (MIUR), Gran Sasso Center for Astroparticle Physics (CFA), CETEMPS
Center of Excellence, Ministero degli Affari Esteri (MAE), Italy; Consejo Nacional de Ciencia y Tecnología (CONACYT), Mexico; Ministerie van Onderwijs, Cultuur en Wetenschap, Nederlandse Organisatie voor Wetenschappelijk Onderzoek (NWO), Stichting voor Fundamenteel Onderzoek der Materie (FOM), Netherlands; National Centre for Research and Development, Grants No. ERA-NET-ASPERA/01/11 and No. ERA-NET-ASPERA/02/11, National Science Centre, Grants No. 2013/08/M/ST9/00322, No. 2013/08/M/ST9/ 00728 and No. HARMONIA 5-2013/10/M/ST9/00062, Poland; Portuguese national funds and FEDER funds within Programa Operacional Factores de Competitividade through Fundação para a Ciência e a Tecnologia (COMPETE), Portugal; Romanian Authority for Scientific Research ANCS, CNDI-UEFISCDI partnership projects Grants No. 20/2012 and No. 194/2012, Grants No. 1/ASPERA2/2012 ERA-NET, No. PN-II-RU-PD2011-3-0145-17 and No. PN-II-RU-PD-2011-3-0062, the Minister of National Education, Programme Space Technology and Advanced Research (STAR), Grant No. 83/2013, Romania; Slovenian Research Agency, Slovenia; Comunidad de Madrid, FEDER funds, Ministerio de Educación y Ciencia, Xunta de Galicia, European Community 7th Framework Program, Grant No. FP7-PEOPLE-2012-IEF-328826, Spain; Science and Technology Facilities Council, United Kingdom; Department of Energy, Contracts No. DE-AC0207CH11359, No. DE-FR02-04ER41300, No. DE-FG0299ER41107 and No. DE-SC0011689, National Science Foundation, Grant No. 0450696, The Grainger Foundation, USA; NAFOSTED, Vietnam; Marie Curie-IRSES/ EPLANET, European Particle Physics Latin American Network, European Union 7th Framework Program, Grant No. PIRSES-2009-GA-246806; and UNESCO.
[1] R. U. Abbasi et al. (HiRes Collaboration), Phys. Rev. Lett. 100, 101101 (2008).

[2] J. Abraham et al. (Pierre Auger Collaboration), Phys. Rev. Lett. 101, 061101 (2008); J. Abraham et al. (Pierre Auger Collaboration), Phys. Lett. B 685, 239 (2010).

[3] K. Greisen, Phys. Rev. Lett. 16, 748 (1966); G. T. Zatsepin and V. A. Kuzmin, Pis'ma Zh. Tekh. Fiz. 4, 114 (1966); JETP Lett. 4, 78 (1966).

[4] D. Allard, Astropart. Phys. 39-40, 33 (2012).

[5] P. Abreu et al. (Pierre Auger Collaboration), Phys. Rev. Lett. 104, 091101 (2010); A. Aab et al. (Pierre Auger Collaboration), Phys. Rev. D 90, 122005 (2014).

[6] R. U. Abbassi et al. (Telescope Array Collaboration), Astropart. Phys. 64, 49 (2015).
[7] V. S. Berezinsky and G. T. Zatsepin, Phys. Lett. B 28, 423 (1969).

[8] D. Hooper, A. Taylor, and S. Sarkar, Astropart. Phys. 23, 11 (2005); M. Ave, N. Busca, A. V. Olinto, A. A. Watson, and T. Yamamoto, Astropart. Phys. 23, 19 (2005).

[9] K. Kotera, D. Allard, and A. V. Olinto, J. Cosmol. Astropart. Phys. 10 (2010) 013.

[10] D. Seckel and T. Stanev, Phys. Rev. Lett. 95, 141101 (2005).

[11] J. K. Becker, Phys. Rep. 458, 173 (2008).

[12] M. G. Aartsen et al. (IceCube Collaboration), Phys. Rev. Lett. 113, 101101 (2014).

[13] E. Waxman and J. N. Bahcall, Phys. Rev. D 64, 023002 (2001). 
[14] M. G. Aartsen et al. (IceCube Collaboration), Phys. Rev. D 91, 022001 (2015).

[15] B. Baret and V. Van Elewyck, Rep. Prog. Phys. 74, 046902 (2011).

[16] J. Abraham et al. (Pierre Auger Collaboration), Nucl. Instrum. Methods Phys. Res., Sect. A 523, 50 (2004).

[17] J. Abraham et al. (Pierre Auger Collaboration), Nucl. Instrum. Methods Phys. Res., Sect. A 613, 29 (2010).

[18] J. Abraham et al. (Pierre Auger Collaboration), Phys. Rev. Lett. 100, 211101 (2008); J. Abraham et al. (Pierre Auger Collaboration), Phys. Rev. D 79, 102001 (2009); P. Abreu et al. (Pierre Auger Collaboration), Astrophys. J. Lett. 755, L4 (2012).

[19] P. Abreu et al. (Pierre Auger Collaboration), Phys. Rev. D 84, 122005 (2011).

[20] R. Fisher, Ann. Eugenics 7, 179 (1936).

[21] J. L. Navarro, Ph.D. thesis, University of Granada, Spain, 2012.

[22] J. Conrad, O. Botner, A. Hallgren, and C. Pérez de los Heros, Phys. Rev. D 67, 012002 (2003).

[23] G. J. Feldman and R. D. Cousins, Phys. Rev. D 57, 3873 (1998).

[24] G. Corcella, I. G. Knowles, G. Marchesini, S. Moretti, K. Odagiri, P. Richardson, M. H. Seymour, and B. R. Webber, J. High Energy Phys. 01 (2001) 010.
[25] S. Jadach, Z. Wąs, R. Decker, and J. H. Kühn, Comput. Phys. Commun. 76, 361 (1993).

[26] A. Cooper-Sarkar and S. Sarkar, J. High Energy Phys. 01 (2008) 075.

[27] S. J. Sciutto, arXiv:astro-ph/9911331, http://www2.fisica .unlp.edu.ar/auger/aires/ppal.html.

[28] P. Billoir, Astropart. Phys. 30, 270 (2008).

[29] http://geant4.cern.ch/.

[30] L. A. Anchordoqui, J. L. Feng, H. Goldberg, and A. D. Shapere, Phys. Rev. D 66, 103002 (2002).

[31] P. W. Gorham et al. (ANITA Collaboration), Phys. Rev. D 85, 049901(E) (2012).

[32] M. G. Aartsen et al. (IceCube Collaboration), Phys. Rev. D 88, 112008 (2013).

[33] K. -H. Kampert and M. Unger, Astropart. Phys. 35, 660 (2012).

[34] M. Ahlers, L. A. Anchordoqui, M. C. Gonzalez-Garcia, F. Halzen, and S. Sarkar, Astropart. Phys. 34, 106 (2010).

[35] J. K. Becker, P. L. Biermann, and W. Rhode, Astropart. Phys. 23, 355 (2005).

[36] O. E. Kalashev, V. A. Kuzmin, D. V. Semikoz, and G. Sigl, Phys. Rev. D 66, 063004 (2002).

[37] V. Berezinsky, A. Z. Gazizov, and S. I. Grigorieva, Phys. Rev. D 74, 043005 (2006). 